\title{
An outflow from the nebula around the LBV candidate S119
}

\author{
K. Weis ${ }^{1,2, \star}$, W. J. Duschl ${ }^{1,2}$, and D. J. Bomans ${ }^{3}$ \\ 1 Institut für Theoretische Astrophysik, Tiergartenstr. 15, 69121 Heidelberg, Germany \\ 2 Max-Planck-Institut für Radioastronomie, Auf dem Hügel 69, 53121 Bonn, Germany \\ 3 Astronomisches Institut, Ruhr-Universität Bochum, Universitätsstr. 150, 44780 Bochum, Germany
}

Received 12 April 2000 / Accepted 8 November 2002

\begin{abstract}
We present an analysis of the kinematic and morphological structure of the nebula around the LMC LBV candidate S 119. On HST images, we find a predominantly spherical nebula which, however, seems to be much better confined in its eastern hemisphere than in the western one. The filamentary western part of the nebula is indicative of matter flowing out of the nebula's main body. This outflow is evidenced by our long-slit echelle spectra. They show that, while most of the nebula has an expansion velocity of $25.5 \mathrm{~km} \mathrm{~s}^{-1}$, the outflowing material reaches velocities of almost $140 \mathrm{~km} \mathrm{~s}^{-1}$, relative to the systemic one. A ROSAT HRI image shows no trace of S 119 and thus no indications of hot or shocked material.
\end{abstract}

Key words. stars: evolution - stars: individual: S 119 - stars: mass-loss - ISM: bubbles - ISM: jets and outflows

\section{Introduction}

Stars with masses in the range of $50-100 M_{\odot}$ and luminosities of $10^{5}-10^{6} L_{\odot}$ populate the upper left end of the Hertzsprung-Russell Diagram (HRD). During their short lives of $\$ 10^{7} \mathrm{yrs}$ they evolve from hot $\mathrm{O}$ stars on the main sequence towards cooler temperatures, first at almost constant luminosities. They soon enter a phase of very strong mass loss of up to $10^{-4} M_{\odot} \mathrm{yr}^{-1}$. This influences their further evolution dramatically: They do not evolve further towards lower temperatures, i.e., towards the red supergiant state, but rather turn in the HRD and become hotter again, albeit later less luminous (e.g., Schaller et al. 1992; Langer et al. 1994).

The region in the HRD where this turn occurs is known to be the domain of the Luminous Blue Variables (LBVs). There exists an empirical limit that separates a region in the HRD into which the most massive stars do not evolve, the so-called Humphreys-Davidson Limit (Humphreys \& Davidson 1979, 1994). Here the stars not only exhibit large continuous mass loss, but at least some of them undergo giant eruptions. Both, the continuous wind and the eruptions lead to a peeling off of the outer parts of the stellar envelope and to the formation of circumstellar $L B V$ nebulae (LBVN; e.g., Nota et al. 1995). Humphreys \& Davidson (1994) classify 32 stars as LBVs and an additional 9 as candidates. 9 of the LBVs and candidate stars

Send offprint requests to: $\mathrm{K}$. Weis,

e-mail: kweis@ita.uni-heidelberg.de

^ Visiting Astronomer, Cerro Tololo Inter-American Observatory, National Optical Astronomy Observatories, operated by the Association of Universities for Research in Astronomy, Inc., under contract with the National Science Foundation. are located in the Milky Way and 10 in the Large Magellanic Cloud (LMC).

$\mathrm{S} 119\left(=\mathrm{Sk}-69^{\circ} 175=\mathrm{HDE} 269687\right)$ is one of the LBVs in the LMC. It was classified as Ofpe/WN9 star by Bohannan $\&$ Walborn (1989). Since the early eighties, there was already the suspicion of a close relation between Ofpe/WN9 stars and LBVs when R127, located again in the LMC and previously classified as Ofpe/WN9 underwent an LBV outburst (Stahl et al. 1983). The evidence for a connection between the two stellar classes has become even stronger since then, as longtime spectroscopic monitoring of LBVs and Ofpe/WN9 stars became available (see, e.g., Stahl \& Wolf 1986; Wolf et al. 1988; Bohannan \& Walborn 1989; Nota et al. 1996; Pasquali et al. 1996).

After discovering a nebula around S 119, Nota et al. (1994) classified the star as an LBV candidate. Their ESO New Technology Telescope (NTT) image shows a nebula of $7^{\prime \prime} \times 9^{\prime \prime}$ size (corresponding to $1.9 \mathrm{pc} \times 2.1 \mathrm{pc}$ for an assumed distance of the LMC of $51.2 \mathrm{kpc}$ ), with a brighter lobe. Their NTT/EMMI spectra indicate an expansion velocity of the $\mathrm{S} 119$ nebula of $\sim 25 \mathrm{~km} \mathrm{~s}^{-1}$, and a ratio of $\mathrm{H}_{\alpha} / \mathrm{N} \sim 1$, leading to $\left[\mathrm{N}_{\mathrm{II}}\right] \lambda 6583 \AA / \mathrm{H}_{\alpha} \sim 0.75$. They derive a radial velocity of the star and of the center of expansion in the range of $100-140 \mathrm{~km} \mathrm{~s}^{-1}$. This casts doubt on $\mathrm{S} 119$ being a member of the LMC the radial velocity of which as derived from $\mathrm{H}_{\mathrm{I}}$ observations (Rohlfs et al. 1984) is typically in the range of $240-300 \mathrm{~km} \mathrm{~s}^{-1}$.

From the line ratio of $\left[\mathrm{S}_{\text {II }}\right] 6716 / 6731 \AA$ Nota et al. (1994) derived an electron density of $n_{\mathrm{e}}=800 \mathrm{~cm}^{-3}$ and - assuming an electron temperature of $T_{\mathrm{e}}=7500 \mathrm{~K}$ - estimated a nebula mass of $\sim 1.7 M_{\odot}$. Similar results for the nebula were reported by Smith et al. (1998). They describe the nebula as 


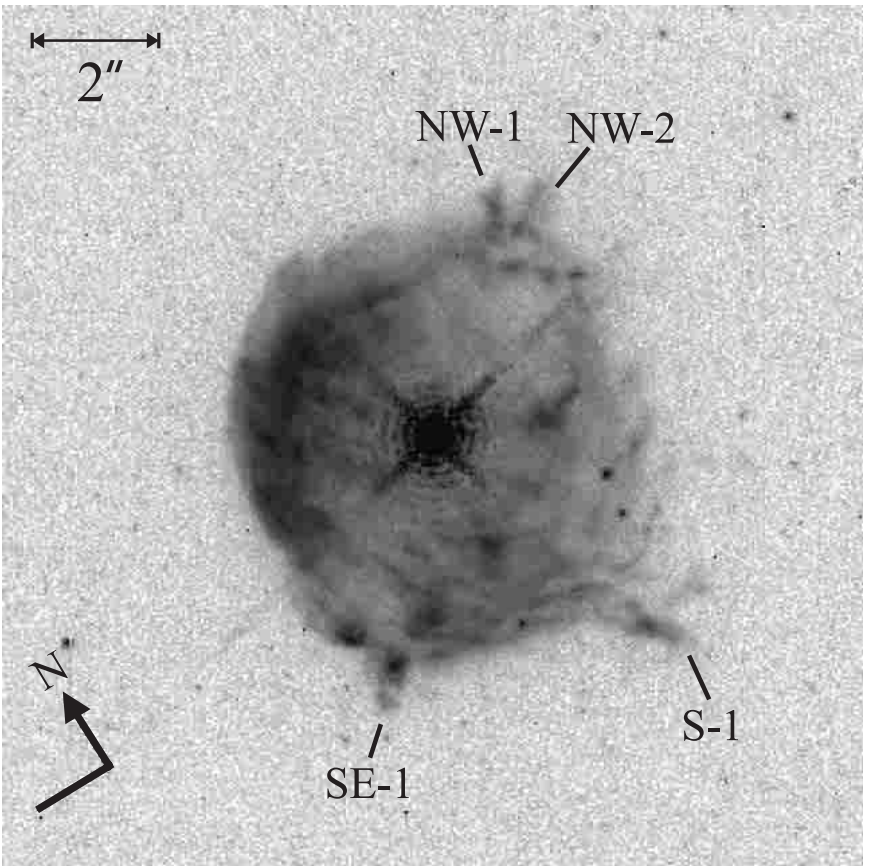

Fig. 1. The F656N HST image of S 119 taken with the PC. The field of view here is $\sim 15^{\prime \prime} \times 15^{\prime \prime}$, and the intensity scaling is logarithmic. A north-east vector indicates the celestial orientation. The filaments described in Sect. 3 are designated as NW-1, NW-2, S-1, and SE-1.

elliptical of size 7 '. $^{\prime} \times 88^{\prime \prime} .6$, with $T_{\mathrm{e}}<6800 \mathrm{~K}$ as estimated from the non-detection of the $\left[\mathrm{N}_{\mathrm{II}}\right] 5755 \AA$ line, and $n_{\mathrm{e}}=680 \mathrm{~cm}^{-3}$. Little reddening and a radial velocity of $v_{\mathrm{rad}}=118 \mathrm{~km} \mathrm{~s}^{-1}$ supports $\mathrm{S} 119$ not being a member of the main body of the LMC. The main stellar parameters of S 119 have been determined by Crowther \& Smith (1997) using two different models to account for the contamination of the nebula in the stellar spectrum: $T_{\text {eff }}=26200 / 27000 \mathrm{~K}, L=5.8 \times 10^{5} / 6.3 \times 10^{5} L_{\odot}$, and $\dot{M}=1.34 \times 10^{-5} / 1.20 \times 10^{-5} M_{\odot} \mathrm{yr}^{-1}$.

In this contribution, we present results of an analysis of the kinematics of the nebula around S 119 and put it for the first time into perspective with the nebula's high-resolution morphology as obtained from Hubble Space Telescope (HST) images. Moreover, we use the non-detection of S 119 and its nebula with the High Resolution Imager (HRI) on board the Röntgensatellit (ROSAT) for determining and discussing upper limits of the X-ray emission.

\section{Observation and data reduction}

\subsection{Imaging}

For the morphological analysis we retrieved from the STScI data archive all images of S 119 taken with the HST Planetary Camera (PC) of the Wide Field Planetary Camera 2 using the $\mathrm{F} 656 \mathrm{~N}\left(\mathrm{H}_{\alpha}\right)$ filter ${ }^{1}$. The exposure times were $500 \mathrm{~s}$ for four images, and 5 and $30 \mathrm{~s}$ for two each. The data were reduced with the standard STSDAS/IRAF routines. In total the four $500 \mathrm{~s}$ exposures were combined and cosmic-ray cleaned. They were

\footnotetext{
1 Program number: 6540; P.I.: Regina Schulte-Ladbeck; dataset names: U4KY0301R...U4KY0308R.
}

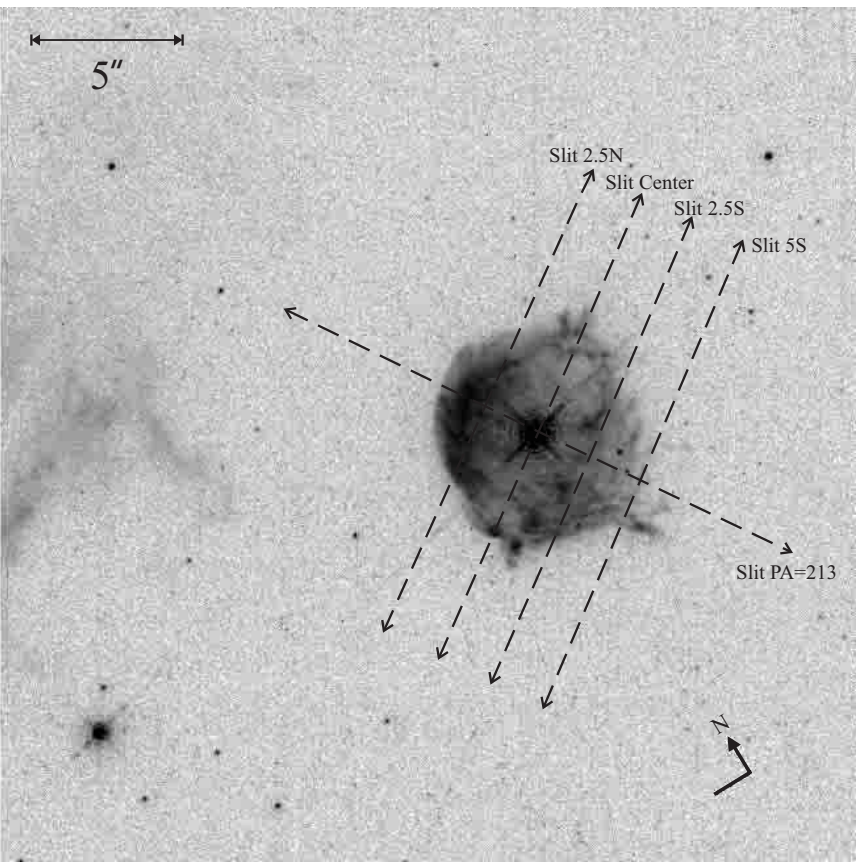

Fig. 2. A larger area $\left(30^{\prime \prime} \times 30^{\prime \prime}\right)$ of the same HST image as in Fig. 1 shows the position and orientation of the slits. In addition to the S 119 nebula emission from a closer $\mathrm{H}$ II region is visible.

not mosaiced since the nebula is fully covered by the PC field of view. The pixel size in the Planetary Camera is $0.0455^{\prime \prime}$ per pixel, the effective resolution about 0 .' 1 . The images were not rotated or binned to make sure to maintain the full resolution. The celestial directions therefore are indicated in the images. The HST system position angle is $148.5^{\circ}$. Figure 1 shows a section of $\sim 15^{\prime \prime} \times 15^{\prime \prime}$ from the reduced PC image which we used for the analysis. The almost full field of view of the PC image is shown in Fig. 2.

\subsection{Long-slit echelle spectroscopy}

For the kinematic analysis of the S119 nebula we obtained high-resolution long-slit echelle spectra with the $4 \mathrm{~m}$-telescope at the Cerro Tololo Inter-American Observatory. For the order selection we replaced the cross-disperser by a flat mirror and inserted a post-slit $\mathrm{H}_{\alpha}$ filter $(6563 / 75 \AA)$. This setup left us with a long-slit vignetted to a length of $\sim 4^{\prime}$ and a spectral range that contained the $\mathrm{H}_{\alpha}$ line and two [N $\mathrm{N}_{\text {II] }}$ lines at $6548 \AA$ and $6583 \AA$. We chose the $791 \mathrm{~mm}^{-1}$ echelle grating and a slit-width of $150 \mu \mathrm{m}$ (corresponding to $1^{\prime \prime}$ ), which lead to an instrumental $F W H M$ at the $\mathrm{H}_{\alpha}$ line of $8 \mathrm{~km} \mathrm{~s}^{-1}$. The data were recorded with the long focus red camera and a $2048 \times 2048$ pixel CCD with a pixel size of $0.08 \AA$ pixel $^{-1}$ along the dispersion and 0 ' 26 pixel $^{-1}$ on the spatial axis. Seeing was $\sim 1$ '.2. Thoriumargon comparison lamp frames were taken for wavelength calibration and geometric distortion correction. Telluric lines visible in the spectra were used to improve the absolute wavelength calibration of which we estimate an accuracy of better than $0.08 \AA$.

We observed at two different position angles (PA) which were nearly perpendicular to each other. Four slit positions 

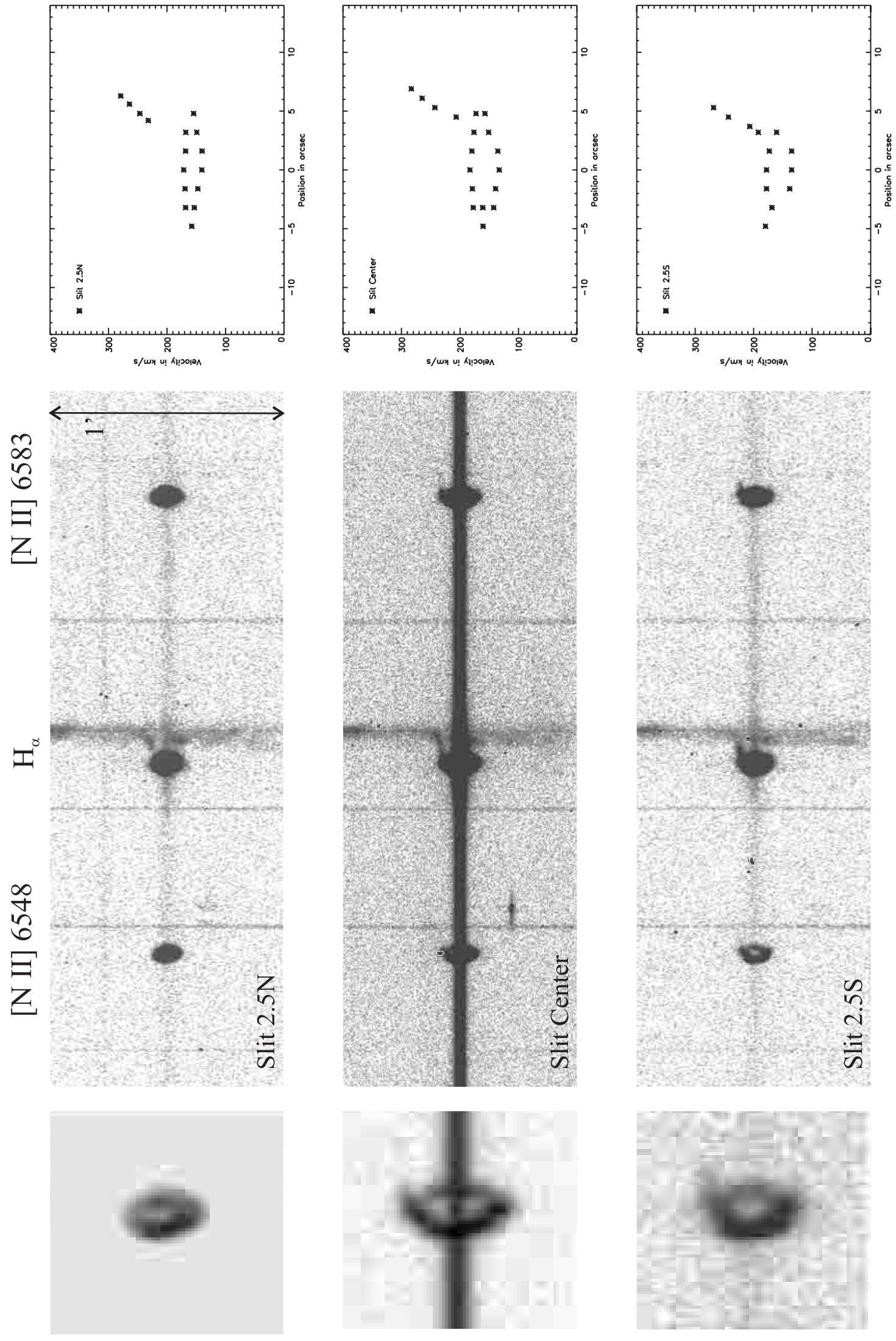

Fig. 3. Echellograms (center column) and corresponding position-velocity diagrams (right column) for the observed slits. Velocity measurements are with respect to the heliocentric system. In the left column an enlargment of the $\left[\mathrm{N}_{\mathrm{II}}\right] \lambda 6583 \AA$ Aine is shown. 

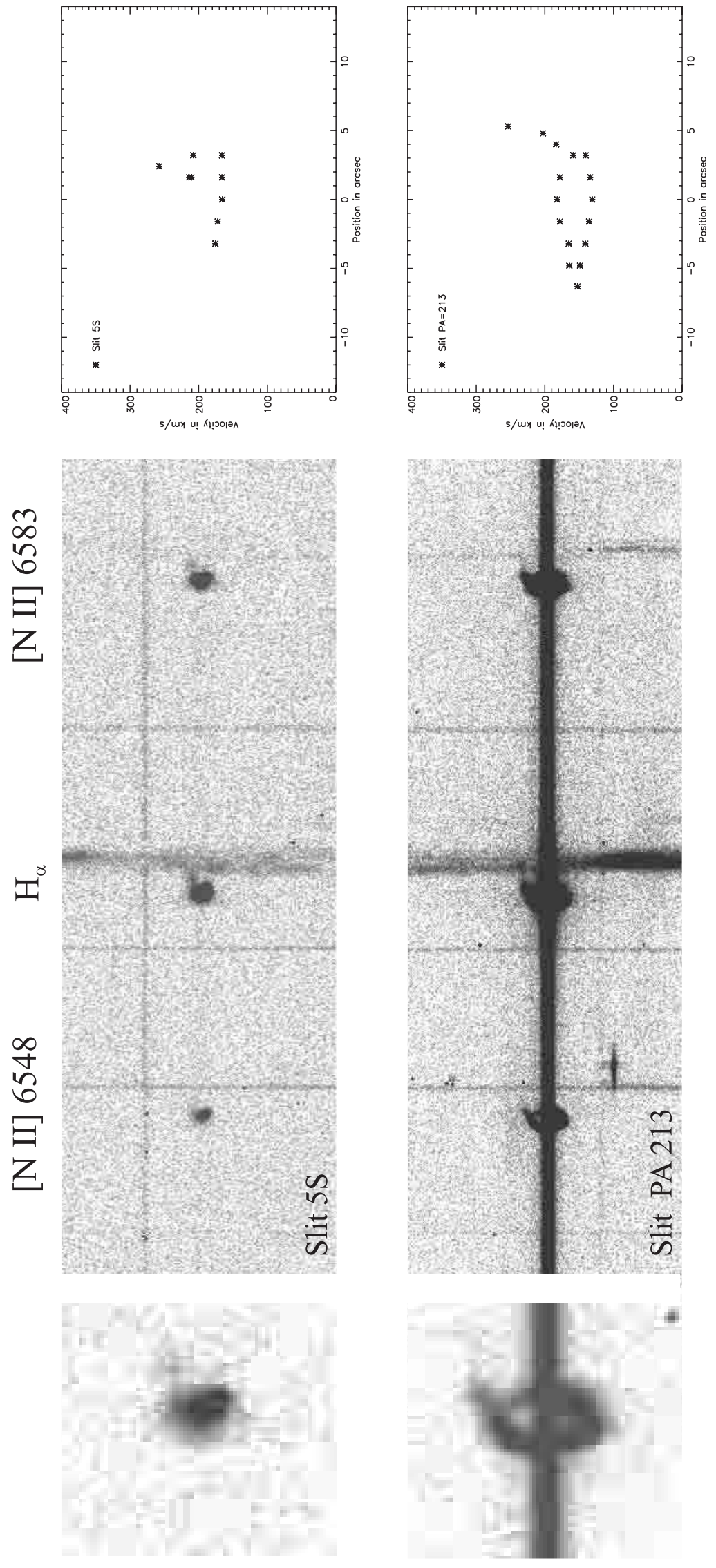

Fig. 4. Echellograms (center column) and corresponding position-velocity diagrams (right column) for the observed slits. Velocity measurements are with respect to the heliocentric system. In the left column an enlargment of the [N II] $] 6583 \AA$ line is shown. 
mapped the nebula with a PA $=125^{\circ}$, one centered on the star (Slit Center), one offset 2.'5 to the north (Slit 2.5N) as well as one each at $2^{\prime \prime} 5$ and $5^{\prime \prime}$ to the south (Slits $2.5 \mathrm{~S}$ and $5 S)$. One additional observation was made with the slit oriented to PA $=213^{\circ}$ and centered on S 119 (Slit PA $=213$ ). Figure 2 shows the slit positions. Figures 3 and 4 summarize our spectroscopic observations. The center column of Figs. 3 and 4 show the echellograms of each slit position we observed, the right column the corresponding position velocity diagrams ( $p v$-diagrams). The echellograms are $54 \AA$ long and extend $1^{\prime}$ in spatial direction, centered on the projected position of the star. The top of the echellograms points towards north-west for $\mathrm{PA}=125^{\circ}$ and towards south-west for PA $=213^{\circ}$. In each echellogram an insert (of $20^{\prime \prime} \times 5 \AA$ ) shows the expansion ellipse of the stronger [ $\mathrm{N}_{\text {II }}$ line $(6583 \AA)$ at a different cut level. The slits are identified according to the nomenclature as introduced above. In the $p v$-diagrams the positions are given as offsets from the projected location of the star. Offsets to the northwest (or, in the case of Slit PA $=213$, south-west) are counted positive. The velocity measurements for the $p v$-diagrams followed from the $\mathrm{H}_{\alpha}$ and the stronger [N $\mathrm{NI}$ line at $6583 \AA$. We estimate an error of the line fits to determine the radial velocities between $\pm 0.5-1 \mathrm{~km} \mathrm{~s}^{-1}$, this is well below the printed symbol size in the $p v$-diagramms. All velocities are with respect to the heliocentric system, unless mentioned otherwise.

\subsection{ROSAT HRI data}

In addition to the optical morphology and the kinematic analysis we looked up ROSAT HRI observations of the S 119 region. The ROSAT satellite was sensitive to X-ray emission between 0.1 and $2.4 \mathrm{keV}$ and its HRI achieves a spatial resolution of $\sim 5^{\prime \prime}$. A 21 ks ROSAT pointing ${ }^{2}$ was retrieved from the MaxPlanck-Institut für Extraterrestrische Physik (MPE) ROSAT data center. After reduction and analysis of the X-ray image with IRAF/PROS ${ }^{3}$, we found no traces of S 119 in the data.

\section{Morphology from the HST image}

Previous images of the nebula around S 119 taken with the NTT show an axisymmetric shell, with a conspicuously brighter lobe at the north east (Nota et al. 1994). The HST F656N images resolve the nebula around S 119 and show that it is approximately spherically symmetric, with an average diameter of 7'.5. Assuming that S 119 is at the same distance as the LMC $(\sim 50 \mathrm{kpc})$, this corresponds to a size of $1.8 \mathrm{pc}$. With the help of the HST especially the small scale structures in and around the nebula of S 119 are clearly detected, which were not seen in previous ground-based images. A large number of these filamemtary structures can be seen in addition to the spherical main body of nebula. The most prominent ones are four filaments extending out of the nebula, two next to each other to the north-west (marked $N W-1$ and $N W-2$ in Fig. 1), one to the

\footnotetext{
${ }^{2}$ P.I.: You-Hua Chu, HRI-pointing: rh600635n00.

3 PROS is developed, distributed, and maintained by the Smithonian Astrophysical Observatory, under partial support from NASA contracts NAS5-30934 and NAS8-30751.
}

south $(S-1)$, and one to the south-east $(S E-1)$. These filaments extend beyond the nebula's main body by $0{ }^{\prime} 96,11^{\prime \prime} 81,0.96$, and 0.68 , respectively. On the west side, between filaments NW-2 and S-1, at the rim of the main body, numerous filaments of comparatively low surface brightness extend beyond it. Here, no clear border of the spherical main body is visible. In this region, some of the filaments appear to be even detached from the main body of the nebula.

In the east, the surface brightness of the nebula is highest, with an $\mathrm{H}_{\alpha}$ surface brightness of $8 \times 10^{-14} \mathrm{erg} \mathrm{cm}^{-2} \mathrm{~s}^{-1} \square^{-\prime \prime}$. This brighter area marks the region seen in seeing-limited NTT images. In the inner part of the nebula the surface brightness is quite low $\left(\sim 4 \times 10^{-14} \mathrm{erg} \mathrm{cm}^{-2} \mathrm{~s}^{-1} \square^{-\prime \prime}\right.$ on average, with some of the filaments being weaker by almost another order of magnitude). There it is far from a homogeneous structure. On the other hand, there are also knots and filaments distributed all over the nebula which are as bright as the eastern part of the nebula. This combination of unevenly high and low surface brightness features give rise to the nebula's patchy appearance.

\section{The kinematic structure of the nebula}

Our high-resolution echelle spectra allow us to analyze the kinematic structure of the S 119 nebula in great detail. At a $F W H M$ resolution of $8 \mathrm{~km} \mathrm{~s}^{-1}$ our observation fully resolve the global structure of the Doppler ellipse of the expansion of the S 119 nebula.

\subsection{The overall expansion}

We find a radial velocity of the center of expansion at $156 \pm$ $2 \mathrm{~km} \mathrm{~s}^{-1}$. This is in agreement with earlier findings that the radial velocity of the $\mathrm{S} 119$ system is well below that of the main part of the LMC. The echellograms show background $\mathrm{H}_{\alpha}$ emission along the entire slit at all positions, which presumably results from an $\mathrm{H}_{\text {II }}$ region within the LMC. The radial velocity of this $\mathrm{H}$ II region of $\sim 264 \mathrm{~km} \mathrm{~s}^{-1}$ is consistent with it being located in the LMC. With respect to this $\mathrm{H}_{\text {II }}$ region, the $\mathrm{S} 119$ nebula moves with about $100 \mathrm{~km} \mathrm{~s}^{-1}$ more along the line of sight.

All spectra passing over the central part of the spherical main body of the nebula show an expansion ellipse as expected for a spherical expansion. We derive a maximum expansion velocity of $25.5 \pm 2 \mathrm{~km} \mathrm{~s}^{-1}$ at the central position in both slits that cross the central star (see $p v$-diagrams for Slit Center and for Slit PA $=213$ at the position $0^{\prime \prime}$ in Figs. 3, 4). As we move away from the geometric center of the nebula, the slits (Slit $2.5 \mathrm{~N}$ and Slit $2.5 \mathrm{~S}$ ) prove the decrease of the expansion, that reduces to $15.5 \mathrm{~km} \mathrm{~s}^{-1}$ and $21.4 \mathrm{~km} \mathrm{~s}^{-1}$ respectively.

We measured sizes of the Doppler ellipses in our echelle data and get diameters of 6.'0 (Slit 2.5N), 9!'1 (Slit Center), and 6.' 8 (Slit 2.5S), respectively. These sizes were determined from the spectra and not from the binned $p v$-diagrams. Given the considerably different spatial resolutions these values agree with those derived from the HST images $\left(5^{\prime \prime} 7,7,8^{\prime \prime} 6\right.$, and 6.'2, respectively). 


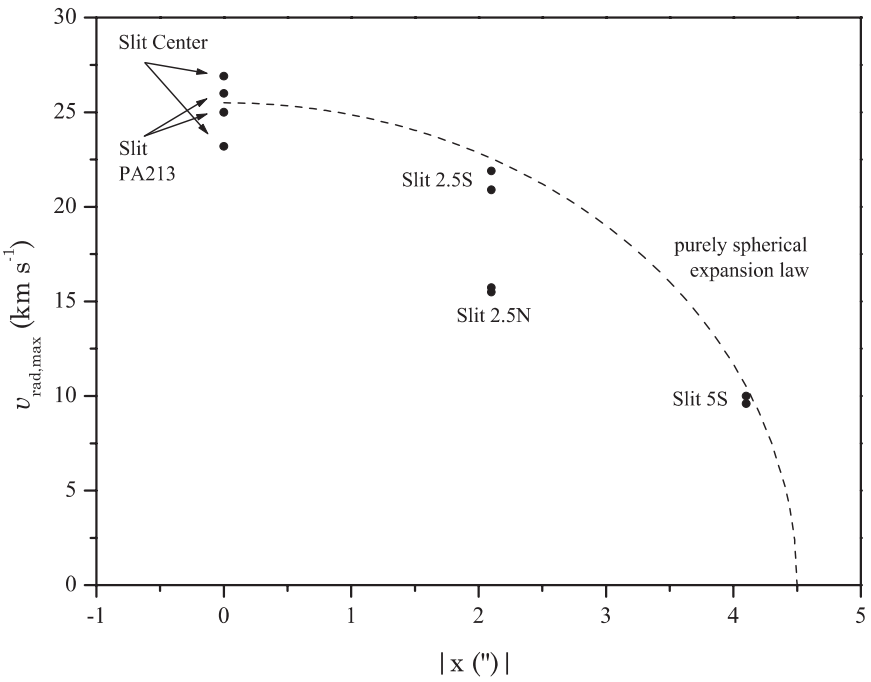

Fig. 5. A position-velocity diagram to compare the measurements with a model of a spherically expanding shell (dashed curve). The errors of the measurement are $\pm(0.5-1) \mathrm{km} \mathrm{s}^{-1}$, and thus of about the size of the dot symbols or smaller.

For a spherical shell of radius $R$ a spherically symmetric expansion with velocity $v_{\exp }$ would lead to an observed radial velocity relative to the systemic velocity

$v_{\text {rad,rel }}^{\text {spher }}(x, y)=v_{\exp }\left(\frac{R^{2}-x^{2}-y^{2}}{R^{2}}\right)^{1 / 2}$

at a location $\{x, y\}$ in a Cartesian coordinate system centered on the star. In the present case, we would assume $R=4$ ". 5 and $V_{\exp }=25.5 \mathrm{~km} \mathrm{~s}^{-1}$ from our data. If we orient the coordinate system such that the $y$-axis is along the slit direction with $y=0$ being the star's projected position onto the slit and $x$ being the offset of the slit with respect to the star, we get for the maximum radial velocities $v_{\text {rad,rel,max }}=v_{\text {rad,rel }}^{\text {spher }}(x=0, y)=$ $25.5 \mathrm{~km} \mathrm{~s}^{-1} \sqrt{1-\left(y / 4^{\prime \prime} 5\right)^{2}}$. In Fig. 5 we give a comparison between the absolute value of the radial velocities (corrected for the systemic velocity) at the star's position projected onto the slit, and the model values. If it were a purely spherical expansion, the measured values should fall onto the dashed model curve). We find a clear deviation from a purely spherical expansion, for instance in the north-south asymmetry in Slits $2.5 \mathrm{~N}$ and $2.5 \mathrm{~S}$.

Both the red-shifted and the blue-shifted components of the expansion ellipse have a $F W H M$ of $0.3-0.5 \AA\left(13-23 \mathrm{~km} \mathrm{~s}^{-1}\right)$, compared to an instrumental $F W H M$ of $8 \mathrm{~km} \mathrm{~s}^{-1}$. The echellograms in Figs. 3 and 4, in particularly the small inserts of the expansion ellipse reveal a clumpy sub-structure. Most prominent in Slit Center, the blue-shifted wing of the ellipse shows brightness variations (at a roughly constant FWHM). These variations are most likely due to the brighter knots identified in the HST image, which were intercepted by the slits. However, due to the large difference in resolution between our spectra and the HST image, no clear identification of the individual knots is possible.

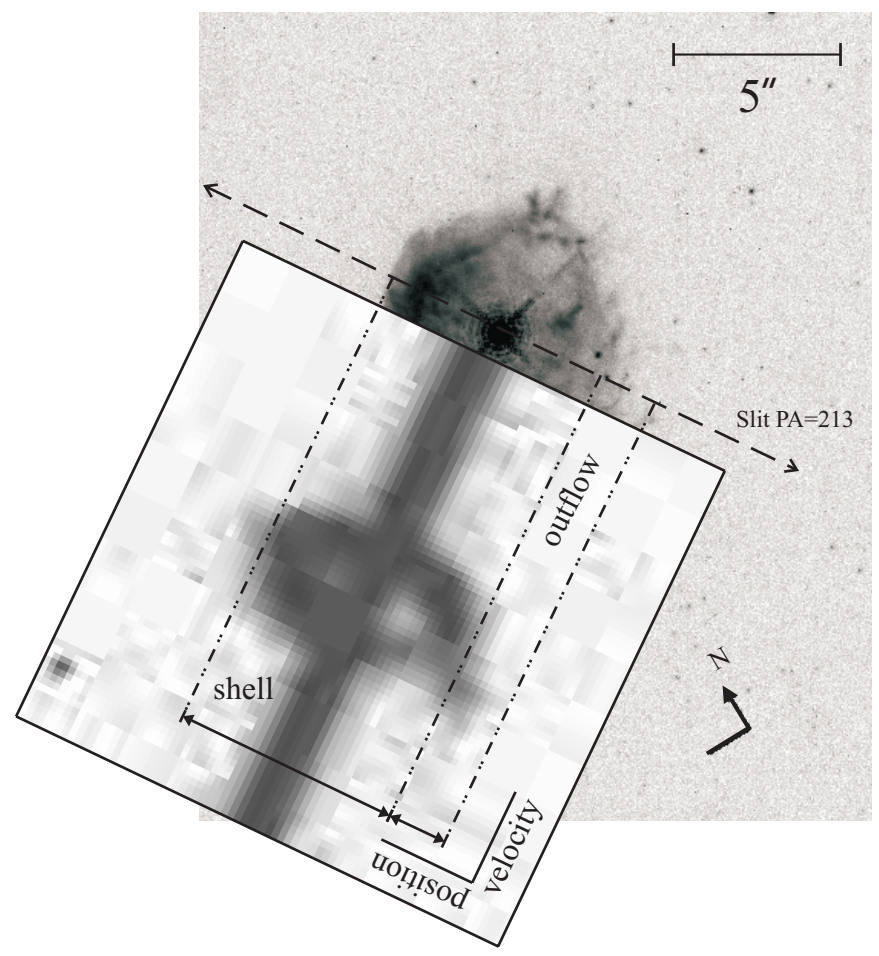

Fig. 6. Overlay of the HST image of S 119 and the spectrum Slit $\mathrm{PA}=213$ around the $[\mathrm{N} \mathrm{II}] \lambda 6583 \AA$ line showing the location of the outflow.

\subsection{The outflow}

In addition to the spherical expansion we find in all spectra clear indications for a much higher velocity expansion structure (for an example, see Fig. 6). It extends spatially from $\sim+4 "$ to $\sim+7^{\prime \prime}$ and in velocity space from the end of the expansion ellipse to more than $280 \mathrm{~km} \mathrm{~s}^{-1}$ redshifted. Only in Slit $2.5 \mathrm{~N}$ this emission feature appears to be detached from the expansion ellipse, and merges with the background $\mathrm{H}_{\alpha}$ emission. To ensure that this high velocity component is part of the S 119 nebula and not a structure in the background emission, we determined its $\left[\mathrm{N}_{\mathrm{II}}\right] \lambda 6583 \AA / \mathrm{H}_{\alpha}$ ratio. If the feature contains $\mathrm{CNO}$ processed material we expect a significantly larger value for an LBV nebula than for an uncontaminated $\mathrm{H}_{\text {II }}$ region. Nebulae around LBVs do have a higher content of nitrogen due to CNO processed material that is mixed into outer regions of the star, which will be ejected and forms the nebula. The higher nitrogen abundance of the nebula can be traced by the $\left[\mathrm{N}_{\text {II }}\right] \lambda 6583 \AA / \mathrm{H}_{\alpha}$ ratio. Typical examples of this ratio for LBVNs are 0.4-0.9 for HR Car (Hutsemékers \& van Drom 1991; Weis et al. 1997), 0.7 for AG Car (Thackeray 1977; Smith et al. 1997), or even as high as 3-7 in $\eta$ Car (Davidson et al. 1982; Meaburn et al. 1987, 1996; Weis et al. 1999). For the spherical nebula around $\mathrm{S} 119$ we derive a ratio of $0.6 \pm 0.1$, for the high velocity component we still find $0.5 \pm 0.1$ (Fig. 7). Both ratios are well within the range found in LBVNs, and are significantly larger than the ratio of the $\mathrm{H}_{\text {II }}$ region in the background of which we observe $\left[\mathrm{N}_{\mathrm{II}}\right] \lambda 6583 \AA / \mathrm{H}_{\alpha}=0.04$. While not far from the limit of our spatial resolution, the combination of spatial and velocity resolution makes it unlikely that our $\left[\mathrm{N}_{\text {II }}\right] \lambda 6583 \AA / \mathrm{H}_{\alpha}$ determination is contaminated. The $\left[\mathrm{N}_{\mathrm{II}}\right] \lambda 6583 \AA$ $/ \mathrm{H}_{\alpha}$ values derived for 


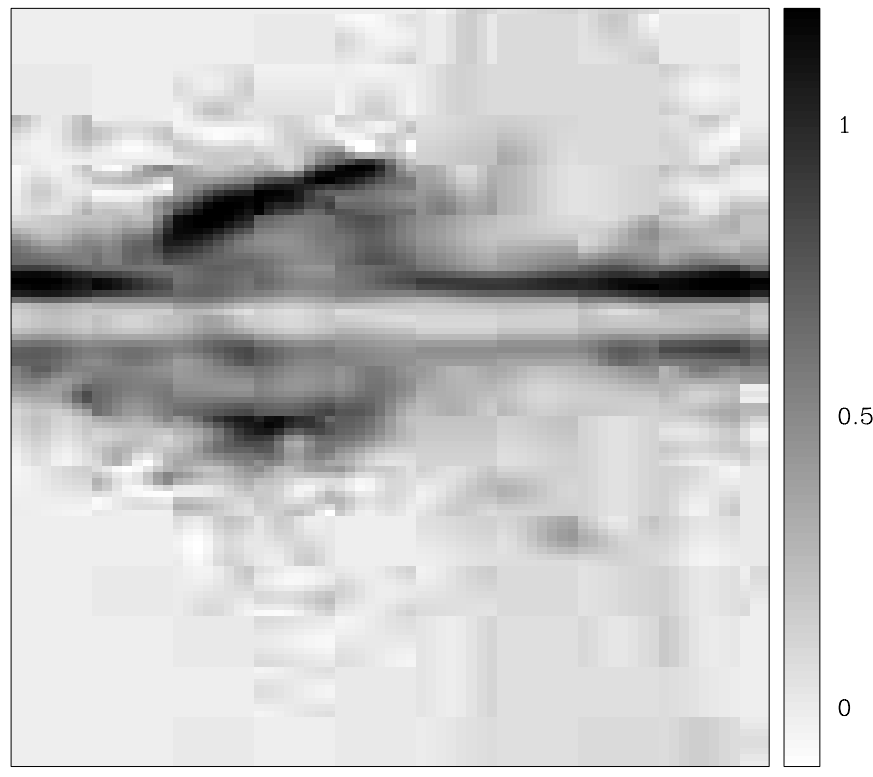

Fig. 7. Ratio map of the $\left[\mathrm{N}_{\mathrm{II}}\right] \lambda 6583 \AA$ and $\mathrm{H}_{\alpha}$ lines of spectrum Slit $\mathrm{PA}=213$. In this gray scale plot of the two-dimensional spectrum we clipped the background so that it is at a near noise-free zero value and slightly smoothed the data with a $3 \times 3$ median to improve the visibility of low surface brightness features. Note the high $\left[\mathrm{N}_{\mathrm{II}}\right] \lambda 6583 \AA$ / $/ \mathrm{H}_{\alpha}$ ratio of the ring and the outflow region compared to the background $\mathrm{H}$ il region.

the nebula agree well with earlier measurements of Nota et al. (1994).

The largest velocity of the high-velocity feature was detected in the Slit Center position with $283.1 \mathrm{~km} \mathrm{~s}^{-1}$, almost $130 \mathrm{~km} \mathrm{~s}^{-1}$ faster than the center of expansion of the central nebula. The velocity of this component increases approximately linearly with distance from the star's projected position. The echellograms do not show any traces of this feature within the Doppler ellipse of the spherical nebula. All these properties strongly point towards this feature being a component of the nebula around S 119 and not a projection effect.

In Fig. 8 we combine the plots of all $p v$-diagrams available to us. A dashed line marks the radial velocity background $\mathrm{H}_{\text {II }}$ region. Both, the spherical expansion of the nebula's main body as well as the high-velocity component are clearly visible. Due to its larger overall offset from the star, in Slit 5S (diamond shape symbols) the outflow seems to set on at a smaller projected offset from the star $\left(+2^{\prime \prime}\right.$ as compared to +4 " for the other slits). This is caused by the effect that the slit intercepts the outflow region earlier.

In Slit 2.5S (square shape symbols) at a projected position of $-5^{\prime \prime}$ another feature appears which moves slightly faster by $\sim 20 \mathrm{~km} \mathrm{~s}^{-1}$. This feature most likely can be assigned to a part of the filament SE-1 identified in Sect. 3.

\subsection{X-ray emission?}

A $21 \mathrm{ks}$ integration with the ROSAT HRI shows no X-ray emission of the nebula. At a distance of $50 \mathrm{kpc}$ and for a Raymond-Smith plasma this corresponds to an upper flux limit of $\sim 1 \times 10^{33} \mathrm{erg} \mathrm{s}^{-1}$, almost independent of assumed

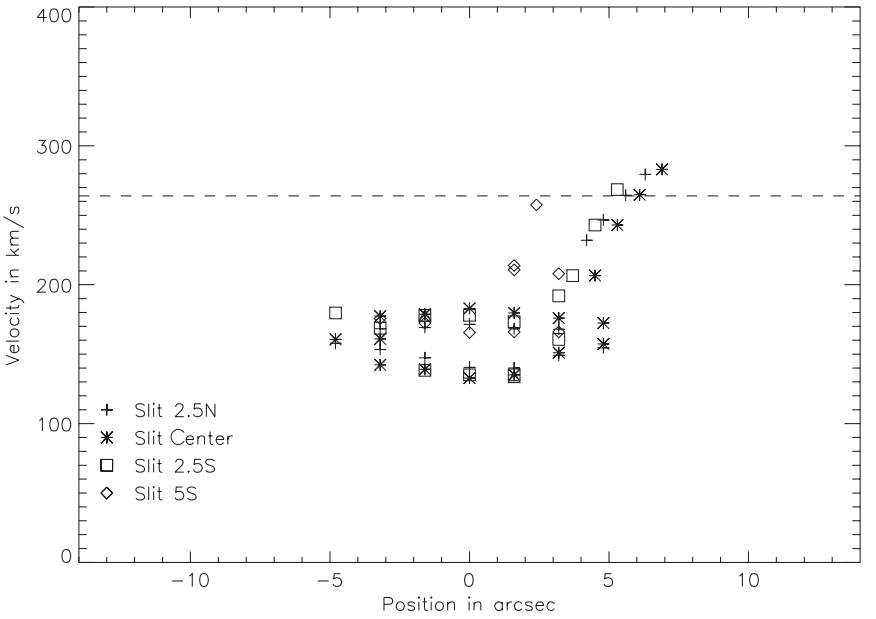

Fig. 8. Combination of all $p v$-diagrams with a $\mathrm{PA}=125^{\circ}$ of Figs. 3 and 4 . The dashed line indicates the radial velocity of the background LMC H II region.

temperatures between 0.1 and $1 \mathrm{keV}$ (see Technical Appendix to the ROSAT Call For Proposals, ROSAT Appendix F). Thus we can only demonstrate that this limit is consistent with the conditions in the S 119 nebula:

- For an energy conserving bubble, for instance, we find an expected X-ray flux of $\sim 2 \times 10^{32} \mathrm{erg} \mathrm{s}^{-1}$ (Weaver et al. 1977; Chu et al. 1995; with a mechanical wind luminosity of $8.1 \times$ $10^{40} \mathrm{erg} \mathrm{s}^{-1}$, an electron density of $700 \mathrm{~cm}^{-3}$, an electron temperature of $7000 \mathrm{~K}$, and an expansion age of $2 \times 10^{4} \mathrm{yr}$ ).

- Moreover, even for the highest outflow velocities $\left(\sim 10^{2} \mathrm{~km} \mathrm{~s}^{-1}\right)$, may lead to post-shock temperatures at a possible interface with the ambient medium of $T_{\mathrm{ps}} \approx 1.3 \times 10^{5} \mathrm{~K}$ and thus give rise to $\mathrm{UV}$ radiation rather than $\mathrm{X}$ rays detectable by ROSAT.

\section{Summary and conclusions}

We have analyzed the morphological and kinematic structure of the nebula around the LBV candidate S 119.

We find that $S 119$ is surrounded by a nebula that represents a spherically expanding shell. On the western rim of this shell, a massive outflow occurs with a much higher velocity than the expanding shell. If one assumes a scenario, in which LBV nebulae are due to wind-wind interactions, the LBVN should be filled with hotter gas, since LBVs-unless in outburst-are hot stars with fast stellar winds. S 119 is a hot star, classified as Ofpe/WN9. The lack of detectable X-rays can therefore be explained by either $(a)$ a relatively old outflow in which the originally hot gas had already sufficient time to cool, or $(b)$ an LBV nebula which was not filled with hot gas in the first place, or (c) a velocity of the outflow which is too small to cause shocks with a sufficiently high post-shock temperature, or - of course - a combination of two of these reasons.

HST images are characterized by the spherical overall appearance of this nebula. The edge of the spherical component is much better defined in the northern and eastern directions where we also find the largest surface brightness. It does not 
exhibit a smooth distribution of surface brightness, but rather is very patchy and shows many filaments and knots, some of which extend - in particular in the western directions - beyond the nebula's main body and occasionally are detached from the main body. This is the reason for the less well defined edge of the nebula in this direction.

High-resolution long-slit echelle spectra show that the spherical component of the nebula is expanding with about $25 \mathrm{~km} \mathrm{~s}^{-1}$ and that the center of expansion is at a radial velocity of $156 \mathrm{~km} \mathrm{~s}^{-1}$. This is remarkable as it is considerably lower than that of the LMC to which-due to its projected position on the sky-the star seems to belong. Both numbers are in good agreement with earlier results (e.g., Nota et al. 1994).

In all spectra a high velocity component is present in addition to the spherical expansion. It corresponds to a relative radial motion of up to $\sim 100 \mathrm{~km} \mathrm{~s}^{-1}$ faster than the spherical main body of the nebula. This high velocity material is concentrated on the western side of the nebula. It is worth noting that on this side the nebula also seems to be somewhat frayed between the filaments NW-2 and S-1 (see Sect. 3). As yet this makes S 119 the only LBV (candidate) nebula with such an outflow. The radial velocities in the outflow increase linearly with distance from the nebula. This Hubble-type velocity law reminds one of the strings found in and around the Homunculus nebula of $\eta$ Car (Weis et al. 1999). However, due to the rather different morphological structure, projection effects (which were ruled out in the $\eta$ Car strings) may play a role in the radial velocity structure in the S 119 outflow. One may, for instance, think of a conical structure with an opening angle that increases outwards. Due to our current lack of understanding of the physical nature of such Hubble type velocity laws, it is not possible to draw firm conclusions on the relation between the two phenomena. The largest radial velocity, relative to the star, amounts to $127 \mathrm{~km} \mathrm{~s}^{-1}$ at a projected distance of 6.' 9 from the star, corresponding to $\sim 1.7 \mathrm{pc}$. One may deduce a minimum dynamical age of $\sim 1.3 \times 10^{4} \mathrm{yrs}$. The dynamical age of the shell assuming a radius of $4 . .5$ and an expansion velocity of $25.5 \mathrm{~km} \mathrm{~s}^{-1}$ amounts than to $\sim 4.2 \times 10^{4}$ yrs. One has to be aware, however, that it depends strongly on the formation mechanism of the outflow how meaningful a dynamical age is. In addition it was shown that the radial velocity of the system seems to deviate from that of the LMC so that the distance to S 119 has to be questioned. This distance on the other hand severely affects the determination of the dynamical age. If S 119 were only $30000 \mathrm{pc}$ away the dynamical age of the nebula would already go down to $\sim 2.5 \times 10^{4} \mathrm{yrs}$. That would than be comparable to the lifetime of the star as an LBV. The dynamical age and the stars position in the HRD, that is being a hot star makes it quite likely that S 119 is already on its way to leave the LBV phase as does He 3-519 (Davidson et al. 1993).

The brightness difference between the west side and the east side (Nota et al. 1994, see also Sect. 3) can most likely be accounted for by the outflow. However, one can only speculate whether the outflow is due to a density gradient in the ambient medium or whether it is caused by asymmetric flows in the S 119 system. Given the sphericity of the nebula's main component we are inclined to hold environment effects responsible for the outflow. This is also consistent with the brightest part of the nebula occurring in the same direction in which the HST image shows ionized diffuse gas which may easily indicate a higher density in that direction.

Acknowledgements. We have made use of the ROSAT Data Archive of the Max-Planck-Institut für extraterrestrische Physik (MPE) at Garching, Germany. Part of the work was carried out on a workstation provided by the Alfried Krupp von Bohlen und Halbach-Stiftung to the ITA. This support is gratefully acknowledged.

\section{References}

Bohannan, B., \& Walborn, N. R. 1989, PASP, 101, 520

Chu, Y.-H., Chang, H.-W., Su, Y.-L., \& MacLow, M. M. 1995, ApJ, 450,157

Crowther, P. A., \& Smith, L. J. 1997, A\&A, 320, 500

Davidson, K., Walborn, N. R., \& Gull, T. R. 1982, ApJ, 254, L51

Davidson, K., Humphreys, R. M., Hajian, A., \& Terzian, Y. 1993, ApJ, 411,336

Humphreys, R. M., \& Davidson, K. 1979, ApJ, 232, 409

Humphreys, R. M., \& Davidson, K. 1994, PASP, 106, 1025

Hutsemékers, D., \& van Drom, E. 1991, A\&A, 248, 141

Langer, N., Hamann, W.-R., Lennon, M., et al. 1994, A\&A, 290, 819

Meaburn, J., Wolstencroft, R. D., \& Walsh, J. R. 1987, A\&A, 181, 333

Meaburn, J., Boumis, P., Walsh, J. R., et al. 1996, MNRAS, 282, 1313

Nota, A., Drissen, L., Clampin, M., et al. 1994, in Circumstellar Media in the Late Stages of Stellar Evolution, ed. R. E. S. Clegg, I. R. Stevens, \& W. P. S. Meikle (Cambridge University Press, Cambridge, UK), 89

Nota, A., Livio, M., Clampin, M., \& Schulte-Ladbeck, R. 1995, ApJ, 448,788

Nota, A., Pasquali, A., Drissen, L., et al. 1996, ApJS, 102, 383

Pasquali, A., Langer, N., Schmutz, W., et al. 1996, ApJ, 478, 340

Rohlfs, K., Kreitschmann, J., Siegman, B. C., \& Feitzinger, J. V. 1984, A\&A, 137, 343

Schaller, G., Schaerer, D., Meynet, G., \& Maeder, A. 1992, A\&AS, 96, 269

Smith, L. J., Stroud, M. P., Esteban, C., \& Vílchez, J. M. 1997, MNRAS, 290, 265

Smith, L. J., Nota, A., Pasquali, A., et al. 1998, ApJ, 503, 278

Stahl, O., \& Wolf, B. 1986, A\&A, 154, 243

Stahl, O., Wolf, B., Klare, G., et al. 1983, A\&A, 127, 49

Thackeray, A. D. 1977, MNRAS, 180, 95

Weaver, R., McCray, R., Castor, J., Shapiro, P., \& Moore, R. 1977, ApJ, 218, 377

Weis, K., Duschl, W. J., Bomans, D. J., Chu, Y.-H., \& Joner, M. D. 1997, A\&A, 320, 568

Weis, K., Duschl, W. J., \& Chu, Y.-H. 1999, A\&A, 349, 467

Wolf, B., Stahl, O., Smolinski, J., \& Casatella, A. 1988, A\&AS, 74, 239 